\title{
UNA CUESTIÓN A DEBATIR. EL NUEVO PERFIL DEL PROTECCIONISMO ESPAÑOL DURANTE LA RESTAURACIÓN
}

\author{
MARCELA SABATÉ SORT \\ EVA PARDOS MARTÍNEZ
}

Universidad de Zaragoza

Cuando el más reciente artículo de Tena sobre indicadores de protección ve la luz en las páginas de esta Revista (otoño-invierno de 1999), nos encuentra trabajando en el vínculo entre apertura externa y crecimiento económico español a largo plazo. Somos por entonces plenamente conscientes de cuán sensibles son los resultados econométricos al indicador de protección considerado, de forma que la lectura de una propuesta de medición alternativa no podía sino despertar nuestro interés. El hecho, además, de sentirnos personalmente aludidos en pasajes concretos del ejercicio, nos movió a poner por escrito ciertas objeciones al respecto.

De una parte, objetamos el uso de desviaciones agregadas como criterio de selección temporal, cuando luego los cálculos de protección se efectúan de manera desagregada. El primer epígrafe, tomando la coyuntura de principios del siglo $\mathrm{xx}$ como escenario, ilustra la debilidad técnica de ese procedimiento y la endeblez de sus resultados. Una objeción adicional surge por el manifiesto desconocimiento de la legislación arancelaria vigente. En el segundo epígrafe se muestra cómo ciertas lagunas de información distorsionan los cálculos del autor, y le confunden, por tanto, a la hora de argumentar sobre su pretendido nuevo perfil del proteccionismo español durante la Restauración. 


\section{LAS VALORACIONES OFICIALES. UN PROBLEMA NO SUPERADO}

A lo largo de esta última década se han sucedido los trabajos de Sabaté (1992, 1996), Prados y Tena (1993) y Tirado (1994), ofreciendo diferentes soluciones a los problemas planteados por la construcción de índices arancelarios para la España de la Restauración. Extensiones de estos trabajos han sido la polémica entre Tena y Tirado (1996) y la evaluación sistemática del origen de las discrepancias hecha por Pardos (1998). Pero es el ejercicio de Tena (1999), el último eslabón de la cadena, el que centra la atención de estas páginas. Empezaremos por el examen de los precios y el criterio de ponderación seleccionados por el autor.

Los precios manejados en Tena (1999) son valores unitarios oficiales y para sortear las posibles distorsiones por falta de fiabilidad de las cifras oficiales, los cálculos se refieren a aquellos años —entre reforma y reforma-, de menores sesgos globales ${ }^{1}$. Esto le lleva a seleccionar los ejercicios $1877,1889,1897,1913$ y 1926, para cada uno de los cuales se calculan tres medias. NT, media que pondera los gravámenes correspondientes a una amplia selección de partidas arancelarias de acuerdo con la participación de cada una de ellas en el total de importaciones del año $t$ considerado; UNT, promedio simple de los anteriores gravámenes; y, en tercer lugar, RNT, que considera los aranceles de un año $t$ determinado (por ejemplo, 1913), empleando para la ponderación la distribución de importaciones del año de referencia anterior (aquí 1897), sobre cuyos precios (valores unitarios), además, se calculan los gravámenes. Finalmente, asumiendo que ninguna medida resulta per se plenamente satisfactoria, construye un cuarto indicador, promedio de los tres anteriores. Dejando a un lado la completa falta de sentido económico del promedio ${ }^{2}$, lo grave es que todas y cada una de las medias que lo componen puedan descalificarse individualmente. ¿La razón? El criterio de selección temporal elegido sólo garantiza una cierta fiabilidad de las cifras oficiales al máximo nivel de agregación, de modo que son perfectamente discutibles los resultados de las medias simples globales y de cualquiera de las ponderadas cuando se desciende en el grado de agregación. Algo relativamente fácil de ilustrar si se toma como referencia, por ejemplo, el cambio arancelario de 1906.

'Según los índices de fiabilidad de las estadísticas comerciales españolas de Prados de la Escosura (1986) y Tena (1992).

${ }^{2}$ Véase al respecto la propia descalificación de Prados y Tena (1993). 
En Tena (1999), la elección de 1897 y 1913 como años con que acotar los cambios introducidos por el Arancel de 1906, responde a que las desviaciones en la cifra total de importaciones (valor oficial - valor estimado/valor estimado $\times 100$ ) fueron del 0,8 y $\times 10,5$ por 100 respectivamente; el que en términos comparados, dentro del período 1891-1922, puedan considerarse años de reducida inexactitud y esto le decidiera a trabajar con ellos, nos parece en principio aceptable. De lo que dudamos es de que trabajando con los valores oficiales de esos años, esté minimizando el impacto de los sesgos en la medición de los niveles desagregados de protección. Para desconfiar, basta plantearse el significado de las desviaciones. El 0,8 por 100 de 1897 , por ejemplo, procede de confrontar las cifras de importación oficial (909,5 millones de pesetas) y estimada $(901,8)^{3}$; la divergencia entre dicho cociente $(1,08)$ y el valor $1-$-que supondría la coincidencia entre cifras oficiales y estimadas- nos da el error 0,8 por 100 . Pero por construcción, este error se trata de un error ponderado: el que deriva de ponderar la diferencia entre valores unitarios oficiales y estimados - más realistas-, por el volumen físico de cada producto (partida) de importación. De esta manera, una desviación global reducida puede encubrir importantes sesgos por productos, que no afloran, bien porque siendo de distinto signo se compensan unos con otros, o simplemente porque no siendo artículos muy relevantes en el total de importaciones, el término de ponderación resulta ínfimo.

No es la primera vez que llamamos la atención sobre el asunto. En Sabaté (1992), (1995), (1996) y (1997) ya se alertaba acerca de que una desviación agregada mínima, nada dice sobre la fiabilidad de las cifras desglosadas. Una advertencia perfectamente aplicable a los años 1897 y 1913. Basta considerar dos productos de importación entonces tan relevantes -en términos físicos y de valor-, como el trigo y el algodón en rama. En 1897 la exageración global, ya se ha dicho, fue del 0,8 por 100; pero en el mismo año, el trigo sufría una exageración de 7 puntos y el algodón se veía afectado por una subvaloración del 5,5 por 100. Más llamativas son las discrepancias en 1913, cuando a una subvaloración global de 10,5 puntos se opone una exageración de 8 de parte del trigo y una subvaloración de 20 puntos en el algodón. Es evidente la escasa relación entre los sesgos por partidas y el agregado que los resume. Por este motivo, decíamos, la media aritmética simple calculada sobre valores oficiales no

${ }^{3}$ Como siempre, los datos de Prados (1986). Debemos además a su amabilidad, los precios unitarios estimados con que revalúa los flujos oficiales de importación en Prados (1981). 
reviste credibilidad alguna; pero tampoco las medias ponderadas sectoriales, si el criterio de selección anual responde a desviaciones en la cifra total de importaciones.

Y en alguna medida debe ser consciente Tena de esta limitación, cuando él mismo aduce como argumento en favor de sus cortes temporales, la moderación del sesgo estimado - disponible también en Prados (1986)para productos primarios y manufacturas. El Cuadro 1 sintetiza la infor-

\section{CUADRO 1}

Sesgos entre cifras oficiales y estimadas

\begin{tabular}{|c|c|c|c|}
\hline & Total & $\begin{array}{l}\text { Productos } \\
\text { primarios }\end{array}$ & $\begin{array}{l}\text { Productos } \\
\text { industriales }\end{array}$ \\
\hline 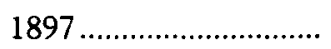 & 0,8 & 5,9 & $-0,6$ \\
\hline $1908 \ldots \ldots \ldots \ldots \ldots \ldots \ldots \ldots \ldots$ & 5,5 & 9,1 & 6,7 \\
\hline 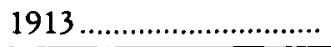 & $-10,5$ & $-9,1$ & $-4,3$ \\
\hline
\end{tabular}

FUENTE: Porcentajes obtenidos a partir de Prados (1986).

mación relativa a tales años: en 1897 una sobrevaloración del 6 por 100 en materias primas y subvaloración del 0,6 en manufacturas; para 1913, subvaloraciones del 9,1 y 4,3 por 100 , respectivamente. Y éste es, en su opinión, un argumento válido, añadido al de las moderadas desviaciones totales, para seleccionar dichos años. La pregunta que se nos ocurre es por qué en lugar de 1913 no ha elegido un año como 1908, con una desviación global más reducida (5,5 por 100), sectorialmente más equilibrada, y sobre todo, con un cambio menos brusco de los sesgos en productos primarios y manufacturas respecto a 1897 . Esta última puntualización carecería de relevancia si el autor no pretendiera hacer comparaciones sectoriales, pero la tiene, y mucha, cuando oponiendo las protecciones relativas (industria/no industria) de 1897 y 1913, concluye por dictaminar el carácter agrario de la reforma de 1906.

Con estas pretensiones, resulta incomprensible que ignore la distorsión introducida por la elección del año 1913, en que el sesgo de productos primarios (comparado con 1897) pasa de una sobrevaluación del 5,6 por 100 a una subvaloración del 10 por 100 , mientras el sesgo sobre manufacturas se mantiene comparativamente estable. En tales condiciones, está claro que la selección de 1913 exagera más que proporcionalmente la pro- 
tección estimada para el conjunto de productos primarios en relación a la industrial, convirtiendo en artificioso el perfil agrario atribuido a la Reforma de 1906. Y nuestra pregunta es: éseguiría detectándose una pérdida de protección relativa de la manufactura si en lugar de tomar 1913 se refirieran los cálculos a 1908, un año aún más legítimo de acuerdo con los propios criterios de Tena (1999)? La respuesta es no.

De acuerdo con el indicador NT calculado por Tena (Cuadro 2), los productos manufacturados ven moderar su protección (del 14,4 a un 14,1

\section{CUADRO 2}

Protección arancelaria ponderada

\begin{tabular}{|c|c|c|c|c|c|}
\hline & & 1897 & 1908 & 1913 & $1913^{\star}$ \\
\hline \multirow[t]{4}{*}{ A) } & Tena & & & & \\
\hline & Productos primarios....................... & 15,1 & & 15,5 & \\
\hline & Productos industriales ................. & 14,4 & & 14,1 & \\
\hline & Industria/No industria .................. & 95,4 & & 91 & \\
\hline \multirow[t]{3}{*}{ B) } & Autores & & & & \\
\hline & Productos primarios........................ & $\begin{array}{l}14,52 \\
162\end{array}$ & $\begin{array}{l}13,69 \\
14,85\end{array}$ & $\begin{array}{l}13,39 \\
15,38\end{array}$ & 16,98 \\
\hline & 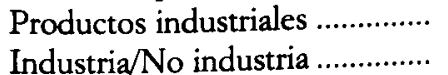 & $\begin{array}{r}16,2 \\
111,6\end{array}$ & & 98,7 & 108,9 \\
\hline
\end{tabular}

* Incluye los datos de la Ley de Comunicaciones Marítimas de 1909.

Fuentes: Tena (1999) y cálculos a partir de las Estadísticas de Comercio Exterior.

por 100 ad valorem), lo que sumado al ligero aumento de la de productos primarios (de 15,1 a 15,5), reduce la protección relativa de la industria, bajando la ratio protección industrial/no industrial de 95,4 a $91^{4}$. Un cambio, por cierto, bastante poco espectacular para unas conclusiones tan contundentes. Pero veamos qué ocurre si introducimos el año 1908 en el

${ }^{4}$ Descalificado por razones ya expuestas el uso de la media simple (UNT), debe repararse en que el año 1908 es un criterio de selección sólo válido, en términos desagregados, para NT. Con relación a la otra media ponderada, RNT, debería buscarse un año entre 1882 y 1891 , de menores sesgos (para productos primarios y elaborados) a los de 1889 . Por ello articulamos la discusión en torno al indicador NT, cuyo perfil, en cualquier caso, mantiene la moderación de la protección manufacturera frente a la agrícola en la coyuntura de principios de siglo. 
cálculo 5 . Lo primero a destacar es el contraste de los porcentajes obtenidos para dicho año y 1913. El cociente protección industrial/no industrial de 1908 queda 10 puntos por encima de la ratio correspondiente a 1913. Algo que obliga a una lectura más ponderada del cambio supuestamente sancionado en la Reforma de 1906, y a moderar, en cualquier caso, las pretensiones del autor sobre haber zanjado la cuestión acerca del carácter agrarista de la ley.

El problema no está únicamente en el año seleccionado, sino en que la falta de conocimiento de la legislación arancelaria del período le lleva a cometer errores de cálculo. Nos referimos al olvido de la famosa Ley de Comunicaciones Marítimas de 1909, cuyos efectos sobre la medición de la protección manufacturera correspondiente a 1913, son totalmente ignorados. A principios del siglo actual, la Reforma Salvador atendió las demandas de las compañías de navegación españolas para reducir el derecho de abanderamiento (arancel) de los buques. Pero la Ley de Comunicaciones Marítimas de 1909, a modo de compensación, elevó las primas percibidas por los armadores. La cuestión no es baladí, pues añadiendo el importe diferencial de las nuevas primas por construcción a la cifra de recaudaciones arancelarias ${ }^{6}$, la protección industrial aumenta del 15,38 a un 16,98 por 100 (Cuadro 2) y la ratio industria/no industria, que es lo que aquí primordialmente interesa, se coloca en 108,98. Un dato asombrosamente cercano al que se obtuvo para 1908, y que abunda en la demanda de moderación para las conclusiones de Tena (1999).

\section{NUEVAS PUNTUALIZACIONES AL TEMA DE LOS ARTÍCULOS DE RENTA}

De acuerdo con este autor, a la pérdida de nitidez del perfil industrial, aparte de la moderación de las barreras impuestas a la manufactura, contribuyó el hecho de que el arancel de 1906 fue más favorecedor de los intereses agrarios que los aranceles precedentes. $\mathrm{Y}$ en este punto, dos son sus argu-

'A efectos de comprobar que no somos nosotros, sino el cambio de año, la única fuente de posibles discrepancias, se acompañan los resultados para 1908 (autores), de los que siguiendo igual proceder, obtuvimos para 1897 y 1913. Que el perfil testimonie, más acentuadamente si cabe, la reducción de la protección relativa industrial (nuestra ratio se precipita de 111,6 en 1897 a 98,7 en 1913), autoriza a argumentar, por comparación, con los resultados de 1908.

- El importe diferencial de las primas en Sabaté (1996, p. 279); el tonelaje importado por clase de buques, en la Estadística de Comercio Exterior de 1913. 
mentos: primero, la marcha de la protección triguera; segundo, en términos más generales, el hecho de que el indicador promedio para alimentos aumente de forma acusada entre 1897 y 1913. Dada la configuración económica del país en la época, creemos que la referencia a uno de los intereses entonces más representativos, el triguero, resulta imprescindible. $Y$ así mismo debe opinar Tena, cuando, a pesar de ponerse en posición un tanto incómoda - descubriendo las debilidades de su método-, no elude el tema. «El régimen arancelario sobre el trigo y sus harinas entre 1892 y 1913 -afirma al respecto-, fue muy cambiante y, por tanto, nuestra elección de fechas anuales para la medición podrían sesgar los resultados sobre la protección relativa del sector» ${ }^{7}$. Sin embargo, los límites de enjuiciar la intensidad de una medida a partir de un solo corte temporal no parecen haberle preocupado mucho hasta ese momento ${ }^{8}$, y nos preguntamos si tan repentino interés guardará alguna relación con el hecho de que obtenga unas protecciones trigueras del 43,7 y 36,4 por 100 cuando ciñe los cálculos a 1897 y 1913. Esto es, que registre una suavización de la protección agraria de difícil engarce en su discurso. Sólo entonces se plantea la necesidad de considerar períodos superiores al año y hacer promedios, 1891-1906 y 1906-1913. Lo realmente paradójico del artificio es que, como tal, hubiese podido evitarse. Bastaba que en lugar de utilizar valores oficiales, hubiera considerado los valores de mercado de Prados (1981), en cuyo caso, habría comprobado que el trigo pasó de soportar un gravamen del 36,6 por 100 en 1897 a otro del 35,5 en 1913. El incómodo desplome de protección triguera que Tena registra en sus cálculos es fruto, simplemente, del escaso realismo de las cifras oficiales de comercio exterior. En este sentido, el propio autor se encarga de demostrar, a precio de incongruencia, el sinsentido de utilizar valores unitarios, por más que en términos globales la desviación entre cifras oficiales y de mercado pueda considerarse mínima.

Pero no hemos terminado. Faltan algunas observaciones a la idea de Tena (1999), según la cual el grupo de alimentos (tomando el promedio de los tres índices) refuerza las defensas frente al exterior entre 1897 y 1913; y esto, aun después de haber eliminado del cómputo aquellos alimentos (coloniales excepto el azúcar) a los que reconoce simple intencionalidad fiscal (Cuadro 3). Cierto es que si medimos las protecciones del grupo de alimentos a partir de la información recogida en la Estadística

7 Tena (1999), p. 601.

8 A pesar de que él mismo es consciente (p. 582) de que las oscilaciones anuales en precios pueden distorsionar la medición igual que un régimen arancelario cambiante. 
CUADRO 3

Protección alimentaria (media de NT, UNT y RNT)

\begin{tabular}{|c|c|c|c|c|c|}
\hline \multirow{4}{*}{ A) } & & Con coloniales & & & $\begin{array}{l}\text { Sin coloniales, } \\
\text { con azúcar }\end{array}$ \\
\hline & & & & & \\
\hline & $1897 \ldots \ldots \ldots \ldots \ldots \ldots$ & 18,9 & & & 21,1 \\
\hline & $1913 \ldots \ldots \ldots \ldots \ldots \ldots \ldots$ & 34,2 & & & 30,7 \\
\hline \multirow{4}{*}{ B) } & & Con coloniales & $\begin{array}{c}\text { Con coloniales } \\
y \text { recargos }\end{array}$ & $\begin{array}{l}\text { Sin coloniales, } y \\
\text { azúcar con recargo }\end{array}$ & $\begin{array}{l}\text { Sin coloniales, } y \\
\text { azucar sin recargo }\end{array}$ \\
\hline & Autores & & & & \\
\hline & $1897 \ldots \ldots \ldots \ldots \ldots \ldots$ & 24,58 & 36,43 & 32,88 & 26 \\
\hline & $1913 \ldots \ldots \ldots \ldots \ldots$ & 39,57 & 39,57 & 33,36 & 33,36 \\
\hline
\end{tabular}

Fuentes: Tena (1999) y cálculos a partir de la Estadística de Comercio Exterior y Sabaté (1996).

de Comercio Exterior, nuestro promedio (que sube del 24 a casi un 40 por 100) dibuja una trayectoria perfectamente comparable a la ofrecida por dicho autor. El problema radica en que para comparar 1897 con 1913, no basta considerar los registros arancelarios.

Minuciosamente explicado por Martín Rodríguez (1982) con relación al azúcar, es generalmente conocido cómo los derechos satisfechos por este producto en frontera no se limitaban a los puramente arancelarios, sino que abarcaban un conjunto de recargos interiores, dependientes de la procedencia, muy discriminatorios con respecto al azúcar peninsular. Una situación que llegó hasta 1899, cuando, perdidas las colonias, no había razón para seguir con el artificio. Se eliminaron entonces los recargos interiores y elevaron, en consecuencia, los aranceles sobre el azúcar extranjero. Y otro tanto ocurrió con el resto de coloniales (los más relevantes, café y cacao), aparentemente sin carga arancelaria cuando procedían de Cuba, Puerto Rico o Filipinas, pero sujetos al mismo sistema de recargos del azúcar. Por consiguiente, parte sustancial del incremento de recaudación arancelario registrado por los coloniales entre 1897 y 1913, no es protección de nuevo cuño, sino mero afloramiento de la protección camuflada en el sistema de recargos interiores. Si incluimos en los cálculos el componente protector de esos recargos, el gravamen sobre alimentos correspondiente a 1897 sube del 24,6 a un 36,4 por 100 ad valorem (Cuadro 3), moderando 
de forma sustancial el abrupto perfil que Tena (1999) da a la evolución de la protección alimentaria entre los aranceles de 1891 y 1906.

Pero sigamos con la cuestión de los alimentos cuya producción nacional es inexistente. En Tena (1999) se eliminan, en efecto, los coloniales"; todos a excepción del azúcar, producto que sí cuenta con producción peninsular. Procediendo de tal modo, concluye que si bien el salto se modera (incluidos los coloniales los porcentajes iban de 18,9 al 34,2 por 100; sin ellos pasan de 21,1 al 30,7 por 100), la imagen de una protección alimentaria reforzada se mantiene, tanto en términos relativos como absolutos. De nuevo pura apariencia. Si sus cálculos para 1897 hubiesen reflejado la tributación real del azúcar antillano - disimulada en el juego de recargos comentado-, el nivel de partida no hubiese sido a buen seguro del 21,1 por 100, sino indudablemente mayor. Así lo prueban nuestros cálculos paralelos. Excluyendo del cómputo a todos los coloniales menos el azúcar -considerando, eso sí, la verdadera discriminación que sufre en frontera durante 1897 - no hay aumento perceptible de protección entre dicho año y 1913, el gravamen se mantiene en torno al 33 por 100. Para que lo hubiese habido, como en Tena (1999), hubiésemos debido ignorar, como él hace, la carga real previa sobre el azúcar cubano (Cuadro 3 ).

\section{REFLEXIONES}

La escasa fiabilidad de las cifras oficiales de importación españolas, sigue pesando como una losa sobre los deseos de desagregación en los cálculos de protección arancelaria nominal. Y aunque hay quienes aceptan la carga y sacrifican detalle o cobertura en la estimación ${ }^{10}$, también hay quien como Tena (1999) no se resigna, y aun a riesgo de que se le achaque amnesia académica ${ }^{11}$, continúa utilizando valores oficiales. Entiéndase que su empeño en suministrar un indicador de protección arancelaria desagregado para el período 1875-1930 - sabedores por experiencia de lo laboriosos y desagradecidos que son los trabajos de medición-, merece todo nuestro respeto. Pero seguimos sin compartir su filosofía. Los contra ejemplos y cálculos aquí presentados muestran sin ambages los problemas que

${ }^{9}$ Celebramos al respecto que se haya finalmente atendido la sugerencia hecha al autor en Sabaté (1996), con relación a los cálculos de Prados y Tena (1993).

${ }^{10}$ En los trabajos de Sabaté (1992, 1996), Tirado (1994) y Gallego (1999).

"Después de tanta denuncia acerca de los peligros derivados de la falta de realismo de los valores oficiales, y tanto esfuerzo -el de Tena (1992), junto a Prados (1981, 1986) —, por salvar a las cifras de comercio exterior de la amenaza. 
conlleva el empleo de valores unitarios oficiales seleccionados con la guía de una desviación agregada, cuando los cálculos de protección bajan al máximo grado de desagregación (media aritmética simple) o utilizan una estructura de ponderación distinta (cualquier ponderación sectorial del tipo industria versus agricultura). Como ilustración de la incongruencia intrínseca al procedimiento, basta recordar el cambio que registra el perfil de protecciones relativas (industria/no industria), si en lugar de tomar los cortes 1897 y 1913, se consideran los años 1897 y 1908 . El cambio es de tal magnitud que quiebra uno de los principales argumentos de su discurso, el de que la Reforma de 1906 vino a reforzar notablemente la protección relativa agraria.

Lo grave es que no sólo la improcedencia del método de selección temporal emborrona el trazo de un nuevo perfil del proteccionismo español (Tena, 1999). Efectos perturbadores tienen también algunos descuidos en materia de legislación arancelaria. Así, la ignorancia de la Ley de Comunicaciones Marítimas, deprime ficticiamente la ratio protección industrial/no industrial de 1913; y un efecto paralelo, aunque afectando al denominador, es el provocado por el olvido de la legislación azucarera en los últimos compases de Imperio. Ambos descuidos bastarían para descalificar la lectura que hace el autor de la Reforma de 1906. Por este motivo, pero sobre todo, por el criticable método de selección temporal empleado, lamentamos concluir que los más recientes esfuerzos de Tena (1999) por homologar clasificaciones y reinterpretar reformas arancelarias, han sido sembrados en baldío.

\section{BIBLIOGRAFIA}

GALLEGO, D. (1999): «Los aranceles y la política de comercio exterior: una aproximación cuantitativa para el caso de los productos agrarios y alimentos (España, 1869-1914)», comunicación presentada al II Encuentro de Economia Aplicada, Zaragoza, junio de 1999.

MarTín Rodríguez, M. (1982): Azúcar y descolonización. Origen y desenlace de una crisis agraria en la vega de Granada. El «ingenio de San Juan», 1882-1904, Granada, Universidad de Granada.

Pardos, E. (1998): «Una revisión de la polémica sobre las mediciones de la protección española durante la Restauración», Actas del Encuentro de Historia Económica, Homenaje a J. Sardá Dexeus, Barcelona.

Prados de la Escosura, L. (1981): «Las estadísticas españolas de comercio exterior 1850-1913: el problema de las valoraciones», Moneda y Crédito, núm. 156, pp. $43-60$. 
- (1986): «Una serie anual del comercio exterior español (1821-1913)», Revista de Historia Económica, núm. 1, pp. 103-150.

Prados de la Escosura, L., y TenA, A., (1993): «Protectionism in Spain 1869-1930», Actas del V Congreso de Asociación de Historia Económica, San Sebastián.

SABATE, M. (1992): La Reforma arancelaria de 1906: la protección legitimada, Tesis Doctoral, Zaragoza.

- (1993): «Tipo de cambio de la peseta y protección a principios de siglo», Revista de Economía Aplicada, núm. 1, pp. 67-86.

- (1995): «La impronta industrial de la reforma arancelaria de 1906», Revista de Historia Industrial, núm. 7, pp. 81-106.

- (1996): El proteccionismo legitimado. Política arancelaria española a comienzos de siglo, Madrid, Civitas.

- (1997): «Las estadísticas españolas de comercio exterior y el cálculo de protecciones arancelarias», Actas del VI Congreso de la Asociación de Historia Económica, Gerona.

Serrano SAnz, J. M. (1991): «La Renta de Aduanas en España (1849-1935)», Hacienda Pública Española, núm. 1, Monografias, pp. 107-119.

TENA, A. (1992): Las estadísticas bistóricas del comercio internacional: fiabilidad y comparabilidad, Madrid, Banco de España.

- (1999): «Un nuevo perfil de proteccionismo español durante la Restauración (1875-1930)», Revista de Historia Económica, núm. 3, pp. 579-621.

TENA, A., y TIRADO, D. A. (1996): «Protección arancelaria en la Restauración. Un debate Antonio Tena-Daniel Tirado», Revista de Economia Aplicada, núm. 11, pp. 135-150.

TIRADO, D. A. (1994): «La política arancelaria en la Restauración. Nuevos indicadores», Revista de Economía Aplicada, núm. 6, pp. 183-203. 